\title{
WZ4002, a third-generation EGFR inhibitor, can overcome anoikis resistance in EGFR-mutant lung adenocarcinomas more efficiently than Src inhibitors
}

\author{
Yuji Sakuma ${ }^{1}$, Yukiko Yamazaki ${ }^{1}$, Yoshiyasu Nakamura' ${ }^{1}$ Mitsuyo Yoshihara', Shoichi Matsukuma', \\ Haruhiko Nakayama ${ }^{2}$, Tomoyuki Yokose ${ }^{3}$, Yoichi Kameda ${ }^{3}$, Shiro Koizume ${ }^{1}$ and Yohei Miyagi ${ }^{1}$
}

Src has a role in the anoikis resistance in lung adenocarcinomas. We focused on two epidermal growth factor receptor (EGFR)-mutant lung adenocarcinoma cell lines, HCC827 (E746-A750 deletion) and H1975 (L858R + T790M), in suspension to elucidate whether suspended lung adenocarcinoma cells are eradicated by long-term treatment with Src tyrosine kinase inhibitors (TKIs). We also examined metastasis-positive lymph nodes from 16 EGFR-mutant lung adenocarcinoma patients for immunohistochemical expression of mutant-specific EGFR. Almost all suspended HCC827 cells underwent apoptosis after $144 \mathrm{~h}$ of combination treatment with AZD0530, trichostatin A (TSA), and ABT-263, whereas many suspended $\mathrm{H} 1975$ cells survived the treatment. AZD0530 is a Src TKI, TSA is a histone deacetylase inhibitor, and ABT-263 is a Bcl-2 inhibitor. During the therapy, the phosphorylation of EGFR decreased in HCC827 cells and remained stable in $\mathrm{H} 1975$ cells. The phosphorylated EGFR of Src TKI-resistant H1975 cells, as well as HCC827 cells, was completely suppressed by the third generation EGFR TKI, WZ4002. Consequently, both the suspended cell lines were almost completely eradicated within $144 \mathrm{~h}$, with the combined therapy of WZ4002, ABT-263, and TSA. Interestingly, treated suspended cells underwent apoptosis to a greater extent than did adherent cells. Intrasinus floating lung adenocarcinoma cells in the lymph nodes expressed a mutant-specific EGFR. These findings suggest that suspended EGFR-mutant lung adenocarcinoma cells depend significantly more on EGFR activation for survival than attached cells do. The tumor cells circulating in vessels, which express mutant-specific EGFR, would be highly susceptible to the combination therapy of WZ4002, ABT-263, and TSA.

Laboratory Investigation (2012) 92, 371-383; doi:10.1038/labinvest.2011.187; published online 12 December 2011

KEYWORDS: anoikis resistance; EGFR; lung adenocarcinoma; Src; WZ4002

We previously reported that approximately half of the patients with a small ( $\leq 2 \mathrm{~cm}$ in size) but vessel invasionpositive lung adenocarcinoma experience relapse in the form of distant metastasis, even if the tumors were completely resected and diagnosed as pathological stage I. ${ }^{1}$ These clinicopathological data indicate that lung adenocarcinoma cells floating in vessels do not undergo apoptosis. In other words, the cells have acquired the ability to resist anoikis, a type of apoptosis induced by detachment from their extracellular matrix. ${ }^{2}$ Several research groups, including us, have reported that Src, a non-receptor type tyrosine kinase, has a role in the anoikis resistance in lung adenocarcinomas. ${ }^{3-5}$ We have demonstrated that 1) intralymphatic floating lung adenocarcinoma cells observed in primary tumor tissues form compact nests expressing E-cadherin and phosphorylated Src; 2) these two proteins are also expressed in tumor spheroids of four lung adenocarcinoma cell lines (LC-KJ, HCC827, H1650, and H1975) cultured in suspension, the shape of which is similar to intralymphatic tumor cell nests observed in tissues. ${ }^{5}$ We thus consider tumor spheroids of the four cell lines in suspension culture system to be a suitable in vitro model of circulating tumor cells. In addition, we have shown that ABT-263, a potent Bcl-2 inhibitor, ${ }^{6,7}$ significantly enhances the susceptibility for apoptosis in suspended lung

\footnotetext{
${ }^{1}$ Molecular Pathology and Genetics Division, Kanagawa Cancer Center Research Institute, Yokohama, Japan; ${ }^{2}$ Department of Thoracic Surgery, Kanagawa Cancer Center Hospital, Yokohama, Japan and ${ }^{3}$ Department of Pathology, Kanagawa Cancer Center Hospital, Yokohama, Japan

Correspondence: Dr Y Sakuma, Molecular Pathology and Genetics Division, Kanagawa Cancer Center Research Institute, 1-1-2 Nakao, Asahi-ku, Yokohama 241-0815, Japan.
}

E-mail: ysakuma@gancen.asahi.yokohama.jp

Received 26 August 2011; revised 17 October 2011; accepted 17 October 2011 
adenocarcinoma cells treated with Src tyrosine kinase inhibitors (TKIs), ${ }^{8}$ as ABT-737, the predecessor of ABT-263, can increase the levels of epidermal growth factor receptor (EGFR) TKI-induced apoptosis in EGFR-mutant lung adenocarcinomas. ${ }^{7,9,10}$ However, the effect of the combination therapy of ABT-263 and a Src TKI on apoptosis in lung adenocarcinoma cells upon detachment was obtained from short-term $(24-48 \mathrm{~h})$ in vitro experiments. ${ }^{5,8}$ We have not yet confirmed whether long-term treatment with a Src TKI and ABT-263 can ultimately eradicate lung adenocarcinoma cells in suspension. Clinical and experimental observations reveal that even highly effective therapies, such as EGFR TKIs for $E G F R$-mutant lung adenocarcinomas cannot eradicate tumor cells. ${ }^{11,12}$ Also, Abl TKIs for chronic myelogenous leukemia (CML) with the $\mathrm{Bcr}-\mathrm{Abl}$ oncogene are not able to eradicate tumor cells, but histone deacetylase (HDAC) inhibitors can eliminate CML stem cells in combination with the Abl TKI, imatinib. ${ }^{13}$ This indicates that a subset of tumor cells can survive treatment with molecularly targeted drugs against the addicting oncogene product through various genetic and/or epigenetic mechanisms. These observations led us to surmise that a subpopulation of lung adenocarcinoma cells in suspension should survive the combination therapy with a Src TKI and ABT-263, but may not last with a combination treatment of a Src TKI, a Bcl-2 inhibitor, and an HDAC inhibitor.

Two EGFR-mutant lung adenocarcinoma cell lines (HCC827 and H1975) cultured in either monolayer ${ }^{14,15}$ or suspension $^{5}$ show different sensitivity to Src TKI treatment. The difference may be because of the fact that only H1975 cells have a point mutation of T790M in exon 20, which confers resistance to first-generation EGFR TKIs such as gefitinib and erlotinib; HCC827 cells, which are sensitive to Src or EGFR TKIs, harbor a deletion mutation in exon 19 (del E746-A750). H1975 cells, which considerably resist Src or EGFR TKI therapy, contain two point mutations of T790M in exon 20 and L858R in exon 21 in the EGFR gene. ${ }^{5,11,14,15}$ The EGFR T790M mutation may have a key role in the decreased susceptibility to Src TKIs in H1975 cells, because mutant EGFR and Src interact with each other. ${ }^{16}$ In this study, we focused on the two EGFR-mutant lung adenocarcinoma cell lines to elucidate to what extent acute and chronic combination therapy with a Src TKI, a Bcl-2 inhibitor, and an HDAC inhibitor, or an EGFR TKI, a Bcl-2 inhibitor, and an HDAC inhibitor, can induce apoptosis in the two cell lines in suspension. We used AZD0530 as the Src TKI, ABT-263 as the Bcl-2 inhibitor, trichostatin A (TSA) as the HDAC inhibitor, and WZ4002 for the EGFR TKI for 24-144 h on HCC827 and H1975 cell lines. We also analyzed whether these combination treatments have the capability of eradicating these suspended carcinoma cell lines.

\section{MATERIALS AND METHODS}

The experimental procedures were approved by the Institutional Review Board at the Kanagawa Cancer Center.

\section{Cell Culture and Drugs}

The two lung adenocarcinoma cell lines (HCC827 and NCIH1975) used in this study were obtained and maintained as previously described. ${ }^{5}$ Cells were grown to subconfluence in 94-mm tissue culture dishes (Greiner Bio-One, Tokyo, Japan) and then, were trypsinized with $0.05 \%$ trypsin/0.02\% EDTA. The cells were then seeded on 90-mm low cell-binding dishes (Nalge Nunc International KK, Tokyo, Japan) for suspension culture or the above-mentioned tissue culture dishes for monolayer culture, and were then cultured at $37^{\circ} \mathrm{C}$ in a $5 \%$ $\mathrm{CO}_{2}$ humidified incubator. The cells cultured on low cellbinding or conventional culture dishes for 24 or $48 \mathrm{~h}$ were then treated with the small-molecule inhibitor(s), as described below, for another $24,48,72$, or $144 \mathrm{~h}$. After $96 \mathrm{~h}$ of plating, the media were removed and replaced with fresh media containing the same $\operatorname{drug}(\mathrm{s})$. The cells treated for $144 \mathrm{~h}$ in suspension were then plated on conventional culture dishes in fresh media without drugs and were allowed to adhere to the surface of the dishes for $24 \mathrm{~h}$ to observe to what degree tumor cells survived the long-term $(144 \mathrm{~h})$ treatment. Media were then removed, and the cells were washed with phosphate-buffered saline and then fixed for $1 \mathrm{~min}$ with $10 \%$ buffered neutral formaldehyde, followed by $99 \%$ methanol for another $24 \mathrm{~h}$. Microscopic images of the cells were taken with a camera and then the cells were stained with Giemsa to underscore the viable cells adhering to the dish. Finally, we counted the number of viable spheroids on the dish to quantify the effect of the long-term therapy on the two cell lines. As a control, cells were treated with the same concentration of dimethyl sulfoxide (Sigma-Aldrich, St Louis, MO, USA). The pharmacological agents used in this study were as follows: two Src family TKIs: AZD0530 (saracatinib; BioVision, Mountain View, CA, USA) or PP1 (Toronto Research Chemicals, North York, Canada); the Bcl-2 inhibitor, ABT-263 (Toronto Research Chemicals); the HDAC inhibitor, TSA (Wako Pure Chemical Industries, Osaka, Japan); and two EGFR TKIs: the first-generation EGFR TKI, gefitinib (Biaffin GmbH \& Co KG, Kassel, Germany) or the third-generation EGFR TKI, WZ4002 (Selleck Chemicals, Houston, TX, USA). WZ4002 has the capability to effectively suppress the phosphorylation of the mutant T790M EGFR, as well as mutant EGFR without the point mutation. ${ }^{17}$ Stock solutions of these drugs were prepared at $10 \mathrm{mM}$ in dimethyl sulfoxide.

\section{The Generation of Src TKI-Resistant H1975 Cells}

H1975 cells were grown to subconfluence in 94-mm tissue culture dishes. The cells then received the combination treatment of PP1 $(5 \mu \mathrm{M})+$ ABT-263 $(1 \mu \mathrm{M})$, which induces apoptosis in $\sim 50 \%$ of suspended H1975 cells in 24 h. ${ }^{8}$ Fresh media containing the drugs were replaced every 3 days. The remaining viable $\mathrm{H} 1975$ cells attached to the dish, decreased in number by about $50 \%$ in response to the treatment within 7 days. However, the cells resumed proliferating even in the presence of the drugs after 2 weeks of drug selection. The cells 
that proliferated to subconfluence in a monolayer culture dish were trypsinized and then resuspended as single cells in fresh media with the drugs. The cells that were seeded again on conventional culture plastic plates were able to thrive in the drug-containing media. We confirmed that the cells survived 20 passages in the presence of the drugs. The cells were termed H1975 Src TKI-resistant (SR) cells in this study.

\section{Western Blotting}

The cells treated with the indicated agent(s) were then lysed in NuPAGE LDS Sample Buffer (Invitrogen, Carlsbad, CA, USA). The whole cell lysates were subjected to SDS-PAGE (NuPAGE $4-12 \%$ Bis-Tris Gel; Invitrogen), followed by blotting with the indicated antibodies, and were detected by the Supersignal West Pico Chemiluminescent Substrate (Thermo Scientific, Rockford, IL, USA). The types and dilutions of primary antibodies used were: anti-poly (ADPribose) polymerase-1 (PARP-1; p116/p85; E78; 1:1000 dilution; Epitomics, Burlingame, CA, USA), anti- $\gamma \mathrm{H} 2 \mathrm{AX}$ (Phospho-Histone H2AX Ser139; 1:1000 dilution; Cell Signaling Technology, Tokyo, Japan), anti-Src (36D10; 1:2000 dilution; Cell Signaling Technology), anti-phospho-Src Family (Tyr416; 1:500; Cell Signaling Technology), antiEGFR (1:1000 dilution; Cell Signaling Technology), antiphospho-EGFR (Tyr1173; 53A5; 1:2000 dilution; Cell Signaling Technology), anti-Histone H3 (1:2000 dilution; Cell Signaling Technology), anti-Acetyl-Histone H3 (Lys14; H3K14 Ac), anti-Akt, anti-phospho-Akt (Ser473), anti-p44/ 42 MAP Kinase (ERK 1/2; 137F5) or anti-phospho-p44/42 MAPK (ERK 1/2; Thr202/Tyr204; D13.14.4E; all at 1:1000 dilution; Cell Signaling Technology), anti-Bim (Y36; 1:1000 dilution; Epitomics), and anti- $\beta$-actin (AC-15; 1:10 000 dilution; Sigma-Aldrich). To evaluate the levels of apoptosis, the band intensity of the pro- and cleaved-forms of PARP-1 was measured on an X-ray film using Image $J$ software. Each experiment was performed in triplicate. PARP-1 is an intracellular 'death substrate' and its cleaved form is used as a representative marker of apoptosis. ${ }^{18}$ We also quantified the band intensity of total and phosphorylated EGFR with the same software.

\section{Another Assessment of Cell Apoptosis}

The two cell lines were seeded on 24-well Cell Tight X plates (Sumitomo Bakelite, Tokyo, Japan) for suspension culture at an initial density of $2.0 \times 10^{5}$ cells per well in $500 \mu \mathrm{l}$ of medium, and allowed to form spheroids for $24 \mathrm{~h}$. The cells were then untreated or treated with the indicated $\operatorname{drug}(\mathrm{s})$ for another $24 \mathrm{~h}$. After $48 \mathrm{~h}$, cell apoptosis was assessed using a Caspase-Glo 3/7 Assay (Promega, Madison, WI, USA) according to the manufacturer's instructions.

\section{The Preparation of Cell Blocks}

Cell blocks of H1975 parental cells and SR cells cultured in suspension for $72 \mathrm{~h}$ were made as previously described. ${ }^{19}$ The cell blocks embedded in agarose were fixed in $10 \%$ buffered neutral formaldehyde and then embedded in paraffin. Immunocytochemical staining of E-cadherin was performed, also as described in the 'Immunohistochemistry' section, to evaluate whether E-cadherin was expressed in both types of spheroids.

\section{Mutational Analysis}

Mutational analyses of the exons 18-21 of the EGFR gene were performed by loop-hybrid mobility shift assays and sequencing as previously described. ${ }^{20}$

\section{Lung Adenocarcinoma Tissues}

We analyzed tumor samples obtained from 16 lung adenocarcinoma patients with lymph node metastasis, who underwent an operation at the Kanagawa Cancer Center Hospital between January 1998 and March 2008. The patients included 6 men and 10 women, with a median age of 62.5 years (range, 52-82 years). Each of the tumors had an activating EGFR gene mutation in exon 19 or 21 (Table 1).

\section{Table 1 Clinicopathological findings, EGFR mutational status, and immunohistochemistry for mutant-specific EGFR in the 16 lung adenocarcinoma patients with lymph node metastasis}

\begin{tabular}{lcclc}
\hline Patient & $\begin{array}{c}\text { Age/ } \\
\text { sex }\end{array}$ & $\begin{array}{c}\text { Lymph node } \\
\text { metastasis }\end{array}$ & $\begin{array}{l}\text { EGFR gene } \\
\text { mutation }\end{array}$ & $\begin{array}{c}\text { Mutant EGFR IHC } \\
\text { (\% positive cells) }\end{array}$ \\
\hline 1 & $66 / \mathrm{F}$ & $\mathrm{pN2}$ & Ex19Del (G3) & 100 \\
2 & $52 / \mathrm{F}$ & $\mathrm{pN2}$ & Ex19Del (G2) & 100 \\
3 & $82 / \mathrm{M}$ & $\mathrm{pN1}$ & Ex19Del (G2) & 90 \\
4 & $70 / \mathrm{F}$ & $\mathrm{pN2}$ & Ex19Del (G3) & 100 \\
5 & $62 / \mathrm{F}$ & $\mathrm{pN2}$ & L858R & 90 \\
6 & $71 / \mathrm{M}$ & $\mathrm{pN2}$ & Ex19Del (G2) & 100 \\
7 & $57 / \mathrm{M}$ & $\mathrm{pN2}$ & L858R & 100 \\
8 & $56 / \mathrm{F}$ & $\mathrm{pN1}$ & L858R & 100 \\
9 & $63 / \mathrm{F}$ & $\mathrm{pN2}$ & Ex19Del (G1) & 30 \\
10 & $59 / \mathrm{M}$ & $\mathrm{pN2}$ & Ex19Del (G2) & 100 \\
11 & $68 / \mathrm{F}$ & $\mathrm{pN2}$ & L858R & 40 \\
12 & $76 / \mathrm{F}$ & $\mathrm{pN1}$ & Ex19Del (G2) & 100 \\
13 & $60 / \mathrm{M}$ & $\mathrm{pN2}$ & L858R & 90 \\
14 & $62 / \mathrm{F}$ & $\mathrm{pN2}$ & L858R & 60 \\
15 & $55 / \mathrm{M}$ & $\mathrm{pN} 1$ & Ex19Del (G2) & 90 \\
16 & $75 / \mathrm{F}$ & $\mathrm{pN2}$ & L858R & 100 \\
\hline & & & & \\
\hline
\end{tabular}

Abbreviations: EGFR, epidermal growth factor receptor; $F$, female; IHC, immunohistochemistry; $M$, male.

a Lymph node metastatic foci were examined for mutant (E746-A750 deletion or L858R)-specific EGFR expression by IHC in each patient. EGFR; Ex19Del, in-frame deletion in exon 19; G1, deletion of nucleotides 2239-2247 and G2248 >C resulted in deletion of amino acids L747-E749 and A750P; G2, deletion of nucleotides 2235-2249 resulted in deletion of amino acids E746A750; G3, deletion of nucleotides 2236-2250 resulted in deletion of amino acids E746-A750; L858R, L858R point mutation in exon 21. 


\section{Immunohistochemistry}

Immunohistochemical staining was carried out on formalinfixed, paraffin-embedded tissue sections of regional lymph nodes that involved metastatic foci for the expression of mutant (E746-A750 deletion or L858R) specific EGFR as previously described. ${ }^{5}$ In addition, immunocytochemical staining was similarly carried out on formalin-fixed, paraffinembedded cell block sections for the expression of E-cadherin. To detect E-cadherin, sections were retrieved by autoclave treatment at $121^{\circ} \mathrm{C}$ for $15 \mathrm{~min}$ in citrate buffer $(\mathrm{pH}$ 6.0). To retrieve two mutant-specific EGFR antigens, sections were autoclaved at $121{ }^{\circ} \mathrm{C}$ for $15 \mathrm{~min}$ in EDTA (pH 9.0). The types and dilutions of primary antibodies used were: anti-Ecadherin (NCH-38; 1:100 dilution; DakoCytomation, Kyoto, Japan), anti-EGFR (E746-A750 deletion mutant specific; 6B6; 1:100; Cell Signaling Technology), and anti-EGFR (L858R mutant specific; 43B2; 1:100; Cell Signaling Technology).

\section{Statistical Analysis}

Differences in rates of apoptosis between cells treated or untreated with the indicated drugs, or between treated cells in suspension or monolayer culture conditions were evaluated by paired $t$-tests. $P$-values of less than 0.05 were considered significant. All statistical calculations were performed with the JMP software system (JMP for Windows version 7; SAS Institute Japan; Tokyo, Japan).

\section{RESULTS}

\section{Short-Term Combination Therapy with AZD0530, ABT-263, and TSA Effectively Induces Apoptosis in HCC827 and H1975 Cells in Suspension}

Although the Src TKI AZD0530 $(0.5 \mu \mathrm{M})$ alone was able to inhibit the Src kinase activity in both the cell lines examined, the induction of apoptosis was not obvious in suspended H1975 cells in terms of PARP-1 cleavage (Figure 1a). Combination therapy with AZD0530 and the Bcl-2 inhibitor ABT263 for $24 \mathrm{~h}$, however, induced apoptosis effectively in the two suspended lung adenocarcinoma cell lines (Figure 1a and $\mathrm{b}$ ). Another DNA damage marker, $\gamma \mathrm{H} 2 \mathrm{AX}$, was induced also in proportion to the degree of apoptosis (Figure 1a). The cells did not undergo marked apoptosis by treatment with
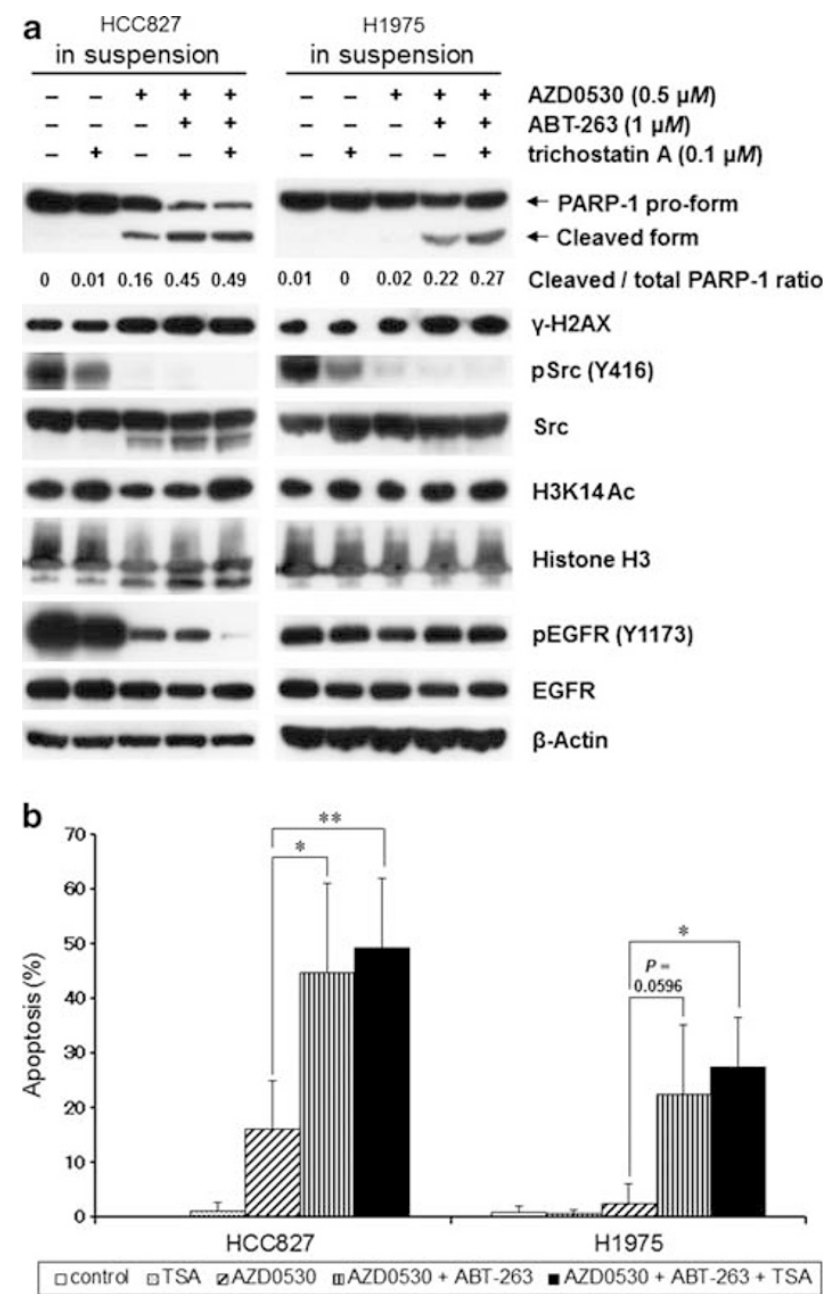

Figure 1 Combination therapy with the Src tyrosine kinase inhibitor (TKI), AZD0530, and the Bcl-2 inhibitor, ABT-263, for $24 \mathrm{~h}$ induces apoptosis effectively in the HCC827 and $\mathrm{H} 1975$ lung adenocarcinoma cell lines in suspension. (a) Western blots examine the effects of AZD0530, ABT-263, with or without the histone deacetylase (HDAC) inhibitor, trichostatin A (TSA), in HCC827 and $\mathrm{H} 1975$ cells. Cells were grown for $48 \mathrm{~h}$ in suspension and then, were treated with the indicated drug(s) for another $24 \mathrm{~h}$. The cleaved/total poly (ADP-ribose) polymerase-1 (PARP-1) ratio determined for each cell line using Image $J$ software is shown also. The values are the means of three independent experiments. (b) The quantification of apoptotic cells assessed by PARP-1 cleavage from each cell line. The means $(n=3)$ and s.d. are plotted. ${ }^{\star} P<0.05 ;{ }^{\star *} P<0.01$.

Figure 2 Combination treatment with AZD0530, ABT-263, and trichostatin A (TSA) for 144h substantially induces apoptosis and almost eradicates suspended HCC827 cells, but not suspended H1975 cells. (a) Western blots examine the effects of the combination treatment of AZD0530, ABT-263, and TSA in suspended or adherent HCC827 cells. HCC827 cells were grown for $24 \mathrm{~h}$ and then incubated with the indicated pharmacological agents for another $72 \mathrm{~h}$. The cleaved/total poly (ADP-ribose) polymerase-1 (PARP-1) ratio determined for each culture condition using Image $\mathrm{J}$ software is also represented. The values are the means of three independent experiments. S, suspension culture; $M$, monolayer culture. (b) Quantification of apoptotic cells assessed by PARP-1 cleavage from HCC827 cells treated in suspension or in a monolayer culture condition. Columns, means $(n=3)$; bars, s.d. ${ }^{* *} P<0.01$. (c) Experimental schema.

(d) Photographs of HCC827 cells. Cells were untreated or treated with the combination therapy for $144 \mathrm{~h}$, were then plated on conventional culture dishes in fresh media without drugs, and were allowed to adhere to the surface of the dishes for another $24 \mathrm{~h}$. Subsequently, the cells were fixed and stained with Giemsa (upper panel), or representative microscopic images were photographed (lower panel) at day 9, as shown in panel c. Original magnification, $\times 200$. (e) Photographs of H1975 cells. Cells were untreated or treated with the combination therapy for $144 \mathrm{~h}$, were then plated on conventional culture dishes in fresh media without drugs, and were allowed to adhere to the surface of the dishes for another $24 \mathrm{~h}$. Subsequently, the cells were fixed and stained with Giemsa (upper panel), or representative microscopic images were photographed (lower panel) at day 9 , as shown in panel c. Original magnification, $\times 40$. 
a

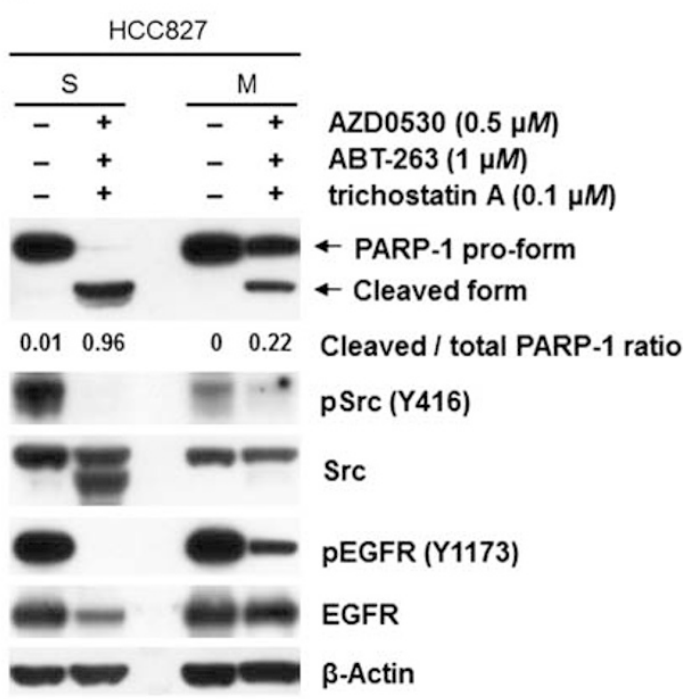

b

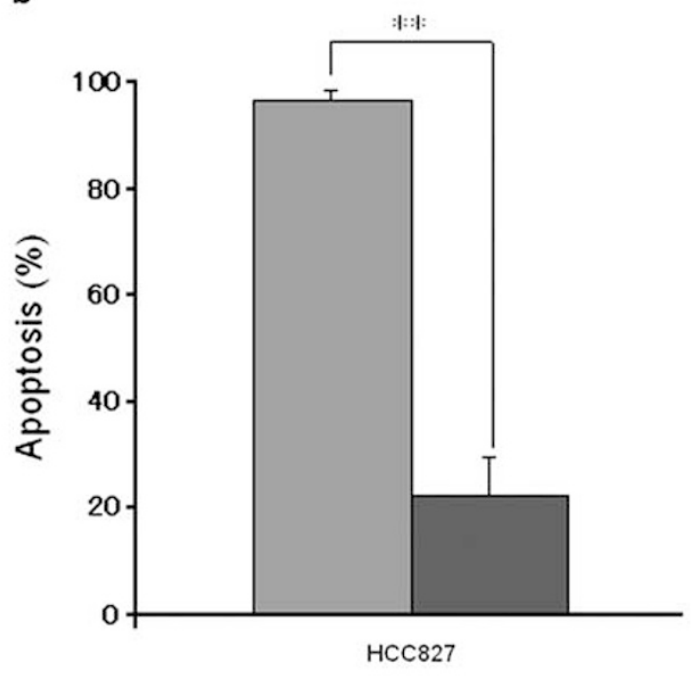

$\square$ Suspension $\square$ Monolayer

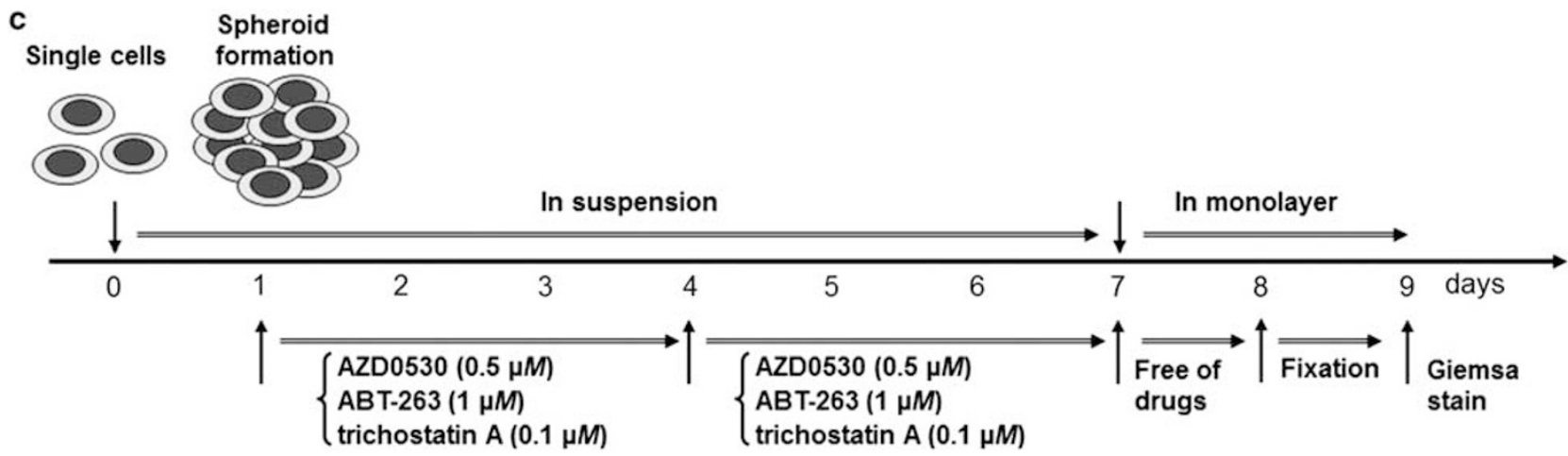

d
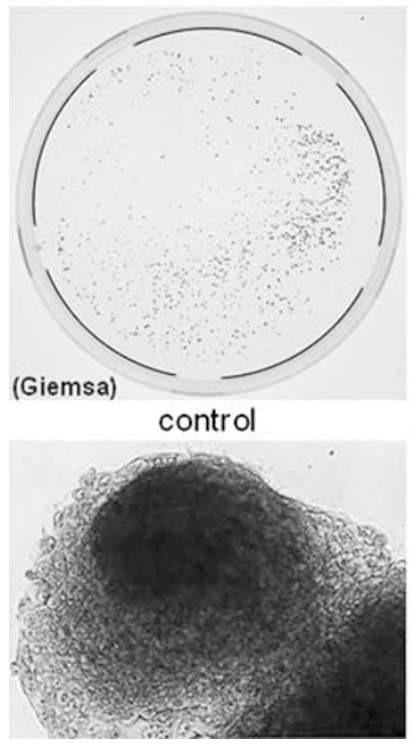

e
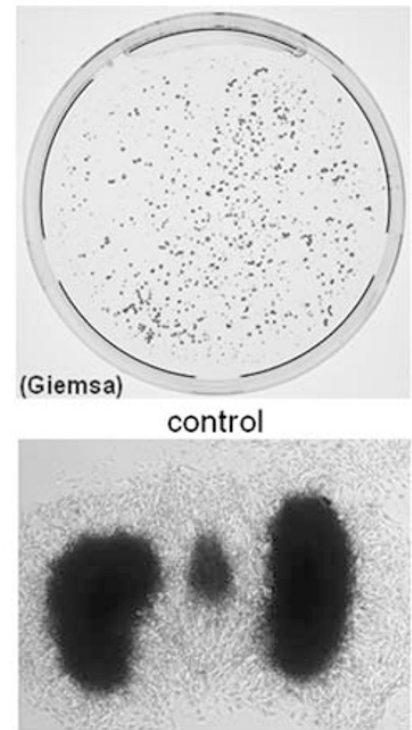

$\mathrm{H} 1975$

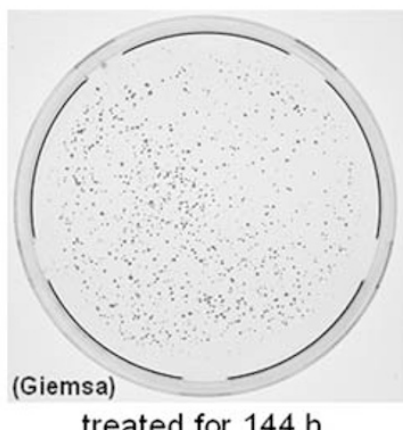
treated for $144 \mathrm{~h}$

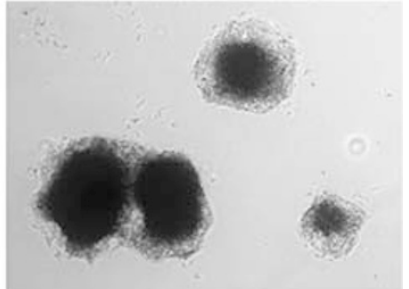


TSA $(0.1 \mu \mathrm{M})$ alone, as tested by the slight induction of acetyl-histone H3K14. The addition of TSA to AZD0530 and ABT-263 treatment slightly enhanced the sensitivity of the cells to apoptosis. However, the difference in apoptosis rates with and without TSA did not reach statistical significance (Figure 1a and $\mathrm{b}$ ). The phosphorylation levels of EGFR dramatically decreased with Src TKI treatment in HCC827 cells, but not in H1975 cells (Figure 1a) as already reported. ${ }^{15}$

\section{Long-Term Combination Treatment with AZD0530, ABT-263, and TSA Substantially Induces Apoptosis and Almost Eradicates Suspended HCC827 Cells, But Many H1975 Cells Cultured in Suspension Survive the Treatment}

More than $90 \%$ of HCC827 cells in suspension underwent apoptosis when they were treated with the three drugs for $72 \mathrm{~h}$ (Figure $2 \mathrm{a}$ and $\mathrm{b}$ ). However, the effect of the combination treatment on HCC827 cells in monolayer, which is in an anchorage-dependent condition, was drastically weaker than that on the cells in suspension (suspension $v s$ monolayer: 96.4 vs $22.4 \%, P=0.0052$; Figure $2 \mathrm{a}$ and $\mathrm{b}$ ). Many tumor spheroids of control HCC827 cells survived in suspension for $144 \mathrm{~h}$ and then adhered to conventional culture dishes, whereas very few treated HCC827 cells were able to survive the therapy and then adhere to the dish (Figure $2 \mathrm{c}$ and $\mathrm{d}$ ). Because the viable HCC827 spheroids after the therapy markedly decreased in number and size, we were not able to observe the spheroids macroscopically (Figure 2d). However, many tumor spheroids of H1975 cells in suspension survived
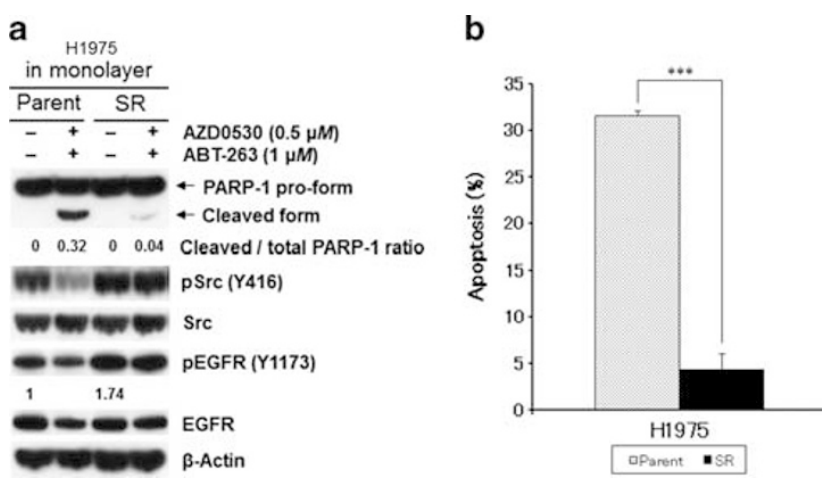

Figure 3 Epidermal growth factor receptor (EGFR) phosphorylation in H1975 SR cells considerably increases compared with parental cells. (a) Western blots examine the effects of the combination treatment of AZD0530 and ABT-263 on H1975 parental or Src tyrosine kinase inhibitor (TKI)-resistant (SR) cells in the monolayer culture condition. Cells were grown for $48 \mathrm{~h}$ and then treated with the indicated drugs for another $48 \mathrm{~h}$. The cleaved/total poly (ADP-ribose) polymerase-1 (PARP-1) ratio determined for each cell line using Image $J$ software is represented. The level of phosphorylated EGFR (pEGFR) relative to total EGFR is also shown. The values are the means of three separate experiments. (b) Quantification of apoptotic cells assessed in terms of PARP-1 cleavage from $\mathrm{H} 1975$ parental or SR cells treated in the monolayer culture condition. Columns, means $(n=3)$; bars, s.d. ${ }^{* *} P<0.001$. the same treatment and subsequently adhered to the dish, although the individual size of the treated spheroids appeared smaller than control cells (Figure 2e). These findings led us to believe that subpopulation of $\mathrm{H} 1975$ cells have the capacity to evade anoikis even in the presence of a Src TKI.

\section{EGFR Phosphorylation in H1975 SR Cells Considerably Increases Compared with Parental Cells}

We established H1975 SR cells that are resistant to the combination treatment of a Src TKI (PP1, $5 \mu \mathrm{M})$ and ABT-263 $(1 \mu \mathrm{M})$. H1975 SR cells showed increased resistance to apoptosis induced by another Src TKI, AZD0530 $(0.5 \mu \mathrm{M})$, and ABT-263 $(1 \mu \mathrm{M})$ as expected (parental cells $v s$ SR cells: 31.5 vs $4.3 \%, P=0.0006$; Figure $3 \mathrm{a}$ and $\mathrm{b}$ ). The combination therapy significantly reduced the phosphorylation levels of Src in H1975 parental cells, but not H1975 SR cells. Also, the expression of phosphorylated EGFR in H1975 SR cells appeared considerably greater than in parental cells (parental cells $v s$ SR cells: 1 vs 1.735, $P=0.0717$; Figure $3 \mathrm{a}$ ). The expression levels of total EGFR were comparable in both parental and H1975 SR cells (parental cells $v s$ SR cells: 1 vs 1.031, $P=0.8299$ ). The upregulation of EGFR kinase activity in SR cells may have a key role in the increased resistance to apoptosis induced by Src TKIs.

\section{H1975 SR Cells Markedly Undergo Apoptosis in Response to Combination Therapy of WZ4002, a Third-Generation EGFR TKI, and ABT-263}

WZ4002 substantially reduced the phosphorylation levels of EGFR in H1975 SR cells, as well as parental cells in almost the same way (Figure 4a). However, the sensitivity of H1975 SR cells to WZ4002-induced apoptosis was considerably lower than parental cells (parental cells $v s$ SR cells for WZ4002 $0.1 \mu \mathrm{M}$ : 19.6 vs $4.1 \%, P=0.0327$; for WZ4002 $1 \mu \mathrm{M}$ : $31.6 v s$ $15.5 \%, P=0.0739$, respectively). The addition of ABT-263 $(1 \mu \mathrm{M})$ significantly enhanced the susceptibility to apoptosis of both the cell lines treated with WZ4002 (Figure 4a and b). Tumor spheroids of H1975 SR cells were markedly fragmented in the presence of WZ4002 and ABT-263 for $24 \mathrm{~h}$ (Figure 4c), and then approximately, half of the treated H1975 SR cells underwent apoptosis (Figure 4a and b). H1975 SR cells were still much more resistant to the combination therapy than parent cells (parental cells $v s$ SR cells for WZ4002 $1 \mu \mathrm{M}+\mathrm{ABT}-2631 \mu \mathrm{M}$ : 59.5 vs $48.0 \%$, $P=0.0151$; Figure $4 \mathrm{~b}$ ). WZ4002 markedly reduced the phosphorylation levels of EGFR in parental cells and SR cells (Figure 4a), suggesting that drug efflux did not account for the increased resistance of SR cells to the EGFR TKI compared with parental cells. As we confirmed that H1975 SR cells retain the same EGFR mutations (T790M + L858R) as the parent cells have (data not shown), they did not arise from contaminating cells. Moreover, H1975 SR cells and parental cells in suspension formed compact multicellular spheroids expressing E-cadherin (Figure 4d), indicating that the epithelial-to-mesenchymal transition, which is often 
a

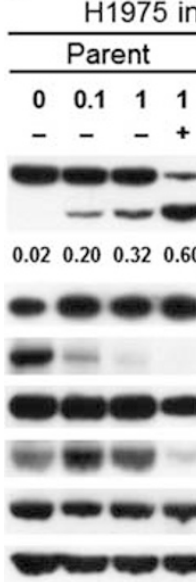

suspension



$\begin{array}{lllll}0 & 0.04 & 0.16 & 0.48 & \text { Cleaved / total PARP-1 ratio }\end{array}$
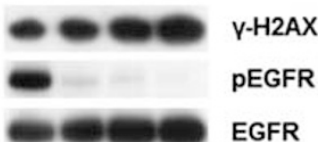

pEGFR (Y1173)

EGFR

pSrc (Y416)

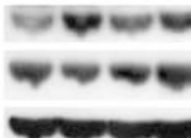

Src

B-Actin b

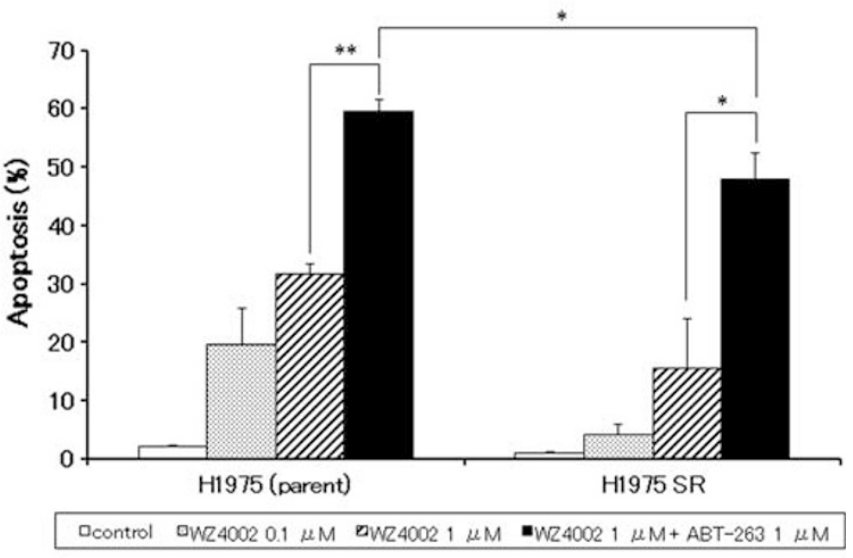

C

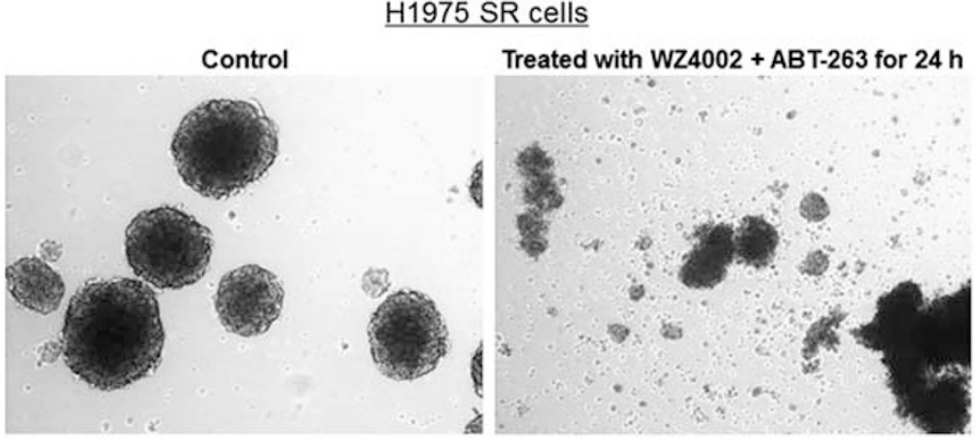

e

H1975 SR

in suspension

- $\quad+\quad$ trichostatin $\mathrm{A}(0.1 \mu \mathrm{M})$

$\leftarrow$ PARP-1 pro-form

$\leftarrow$ Cleaved form

0.020 .02 Cleaved $/$ total PARP-1 ratio

d

H1975 parent

H1975 SR
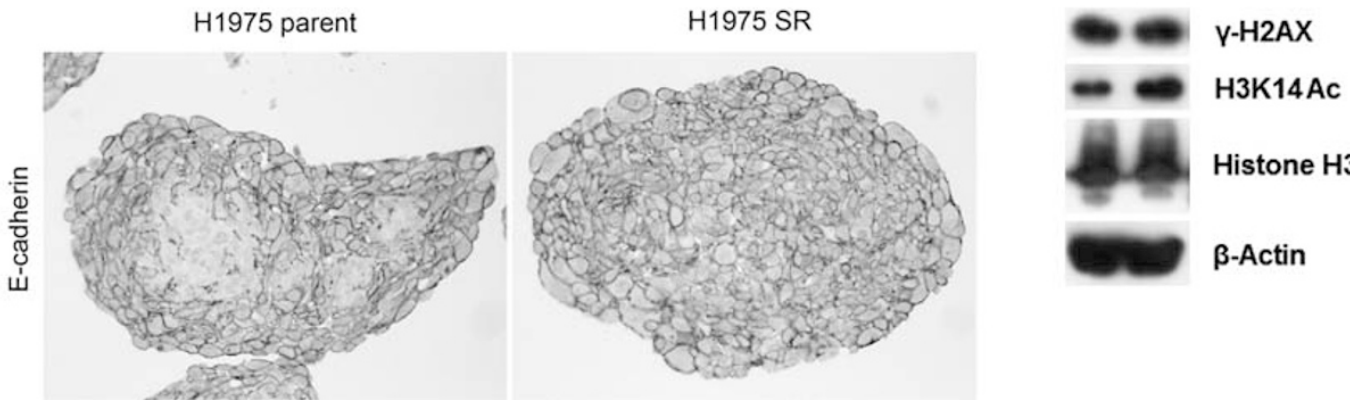

Figure $4 \mathrm{H} 1975$ Src tyrosine kinase inhibitor (TKI)-resistant (SR) cells markedly undergo apoptosis in response to the combination therapy of WZ4002 and ABT-263. (a) Western blots examine the effects of WZ4002 alone or a combination of WZ4002 and ABT-263 in H1975 parental or SR cells in suspension. Cells were grown for $48 \mathrm{~h}$ and then treated with the indicated pharmacological agent(s) for another $24 \mathrm{~h}$. The cleaved/total poly (ADP-ribose) polymerase-1 (PARP-1) ratio determined for each cell line using Image J software is also represented. The values are the means of three independent experiments. (b) Quantification of apoptotic cells assessed by PARP-1 cleavage from each cell line. The means $(n=3)$ and s.d are plotted. ${ }^{*} P<0.05 ;{ }^{*} P<0.01$. (c) Cell morphology of H1975 SR cells. H1975 SR cells were seeded and left in suspension for $48 \mathrm{~h}$, and then untreated or treated with the combination treatment of WZ4002 and ABT-263 for another $24 \mathrm{~h}$ before being photographed. (d) Immunocytochemical analysis of H1975 parental and SR cells for E-cadherin expression. (e) Western blots examine the effects of trichostatin A (TSA) in suspended H1975 SR cells. Cells were grown in suspension for $48 \mathrm{~h}$ and then incubated with or without TSA for another $24 \mathrm{~h}$. The cleaved/total PARP-1 ratio determined using Image J software is also shown. The values are the means of three independent experiments.

associated with acquired drug resistance in EGFR-mutant lung adenocarcinomas, ${ }^{21}$ did not occur in the SR cells. Although EGFR phosphorylation of both the cell lines similarly declined, the increased resistance of H1975 SR cells to WZ4002-induced apoptosis compared with parental cells may be because of transient drug tolerance, based on chromatin modification by histone demethylase KDM5A/Jarid1A proposed by Sharma et al..$^{22}$ Although this ability to tolerate drug exposure seems to be vulnerable to HDAC inhibition, ${ }^{22}$ SR cells did not undergo apoptosis when treated with the HDAC inhibitor, TSA $(0.1 \mu \mathrm{M}$; control $v s$ TSA: $1.9 \%$ vs $1.8 \%$, $P=0.86)$, and the induction of $\gamma \mathrm{H} 2 \mathrm{AX}$ was not observed in either untreated or treated SR cells (Figure 4e). It seems unlikely that the chromatin-mediated reversible drug-tolerant 
state accounts for the resistance to WZ4002-induced apoptosis in H1975 SR cells. However, suspended HCC827 cells were highly sensitive to WZ4002 alone, as well as the combination therapy of WZ4002 and ABT-263, and underwent apoptosis easily with the combination treatment (Supplementary Figure S1a and b).

\section{Suspended H1975 SR Cells and HCC827 Cells are Significantly More Sensitive to EGFR TKI-Induced Apoptosis in Comparison with Adherent Cells}

Approximately 80 and 90\% of H1975 SR and HCC 827 cells, respectively, in suspension underwent apoptosis when the cells were treated with WZ4002 and ABT-263 for $72 \mathrm{~h}$ (Figure $5 \mathrm{a}$ and $\mathrm{b}$ ). Intriguingly, the extent of apoptosis induced by the same treatment in both types of cells in monolayer culture conditions markedly declined compared with that in suspension, although the phosphorylation of EGFR was completely suppressed by the treatment regardless of suspension or monolayer culture conditions (suspension $v s$ monolayer: $81.7 v s$ 47.0\% in H1975 SR cells, $P=0.0046$; 91.6 vs $12.5 \%$ in HCC 827 cells, $P=0.0017$; Figure $5 \mathrm{a}$ and b). The marked difference in the sensitivity to EGFR TKI-induced apoptosis between culture conditions was also observed in gefitinib-treated HCC827 cells (suspension vs monolayer: 86.0 vs 31.2\%, $P=0.0053$; Supplementary Figure S2a and b).

\section{Long-Term Combination Treatment with WZ4002, ABT-263, and TSA Substantially Induces Apoptosis in Suspended H1975 SR Cells and HCC827 Cells to the Level of Almost Eradication}

As treatment with HDAC inhibitors combined with Abl TKIs can eliminate CML stem cells, ${ }^{13}$ we evaluated the effect of the addition of TSA to WZ4002 and ABT-263 treatment on the two cell lines. Short-term $(24 \mathrm{~h})$ treatment with the three drugs induced much more apoptosis in H1975 SR cells than the combined therapy with WZ4002 and ABT-263 in terms of caspase $3 / 7$ activation assay (two drugs $v s$ three drugs: $2.02 \pm 0.06$ vs $2.51 \pm 0.14, P=0.0136$; Figure 6a) and PARP-1 cleavage assay (two drugs vs three drugs: $20.4 \pm 5.0$ vs $33.9 \pm 3.7 \%, P=0.0257$; Figure $6 \mathrm{~b}$ ). For HCC 827 cells treated for $24 \mathrm{~h}$, neither caspase $3 / 7$ activation assay (two drugs $v s$ three drugs: $3.55 \pm 0.28$ vs $3.52 \pm 0.05, P=0.8665$; Figure 6a), nor PARP-1 cleavage assay (two drugs $v s$ three drugs: $94.8 \pm 3.7$ vs $94.6 \pm 3.8 \%, P=0.7943$; Figure $6 \mathrm{~b}$ ) showed any additive or synergistic effect on apoptosis with the addition of TSA. The combined therapies with or without
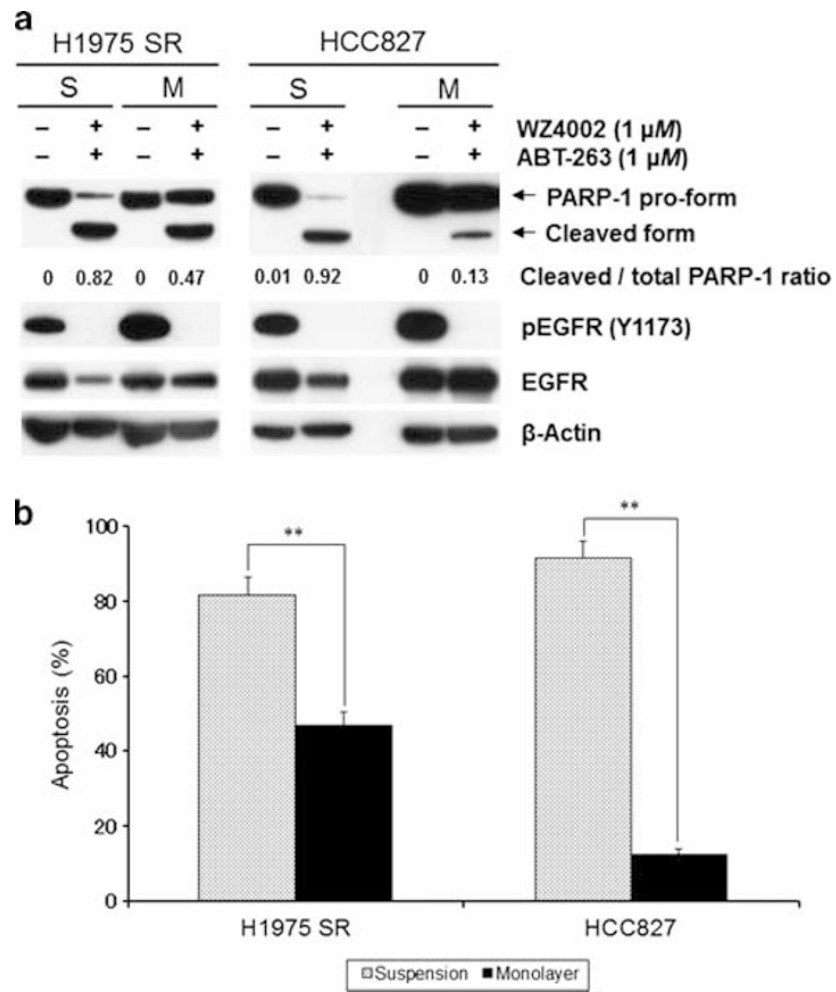

Figure 5 Suspended H1975 Src tyrosine kinase inhibitor (TKI)-resistant (SR) cells and $\mathrm{HCC} 827$ cells are substantially more sensitive to epidermal growth factor receptor (EGFR) TKI-induced apoptosis in comparison with adherent state. (a) Western blots examine the effects of the combination therapy of WZ4002 and ABT-263 on H1975 SR and HCC827 cells cultured in suspension or as a monolayer. Cells were grown for $24 \mathrm{~h}$ and then treated with the indicated drugs for another $72 \mathrm{~h}$. The cleaved/total poly (ADP-ribose) polymerase-1 (PARP-1) ratio determined for each cell line using Image J software is also represented. The values are the means of three independent experiments. S, suspension culture; M, monolayer culture. (b) Quantification of apoptotic cells assessed by PARP-1 cleavage from suspended or adherent H1975 SR and HCC827 cells. Columns, means $(n=3)$; bars, s.d. ${ }^{*} p<0.01$

Figure 6 Long-term combination treatment with WZ4002, ABT-263, and trichostatin A (TSA) substantially induces apoptosis in suspended H1975 SrC tyrosine kinase inhibitor (TKI)-resistant (SR) cells and HCC827 cells to the level of almost complete eradication. (a) Caspase 3/7 activation in H1975 SR cells and HCC 827 cells. Cells were seeded and left in suspension for $24 \mathrm{~h}$, and then untreated or treated with the indicated drug(s) for another $24 \mathrm{~h}$ before being assessed. Caspase activity was evaluated by using the Caspase-Glo 3/7 assay and normalized to a 1.0 arbitrary unit for the mean of three untreated wells. The means $(n=3)$ and s.d are plotted. ${ }^{*} P<0.05$. (b) Western blots examine the effects of the indicated combination treatment in suspended H1975 SR cells and HCC 827 cells. Cells were grown for $24 \mathrm{~h}$ and then incubated with the indicated pharmacological agents for another $24 \mathrm{~h}$. The cleaved/total poly (ADP-ribose) polymerase-1 (PARP-1) ratio determined for each cell line using Image $\mathrm{J}$ software is also represented. The values are the means of three independent experiments. EL, extra long; L, long, S, short. (c) Experimental schema. (d) Photographs of H1975 SR cells and HCC827cells. Cells were untreated or treated with the indicated combination therapy for $144 \mathrm{~h}$, were then plated on conventional culture dishes in fresh media without drugs and were allowed to adhere to the surface of the dishes for another $24 \mathrm{~h}$. Subsequently, the cells were fixed and stained with Giemsa (upper panel), or representative microscopic images were photographed (lower panel) at day 9, as illustrated in panel c. Original magnification, $\times 100$ (low-power view) or $\times 400$ (inset). (e) Quantification of the number of viable spheroids or clusters from each cell line treated at day 9 . The means $(n=3)$ and s.d are plotted. ${ }^{*} P<0.01$. 
a

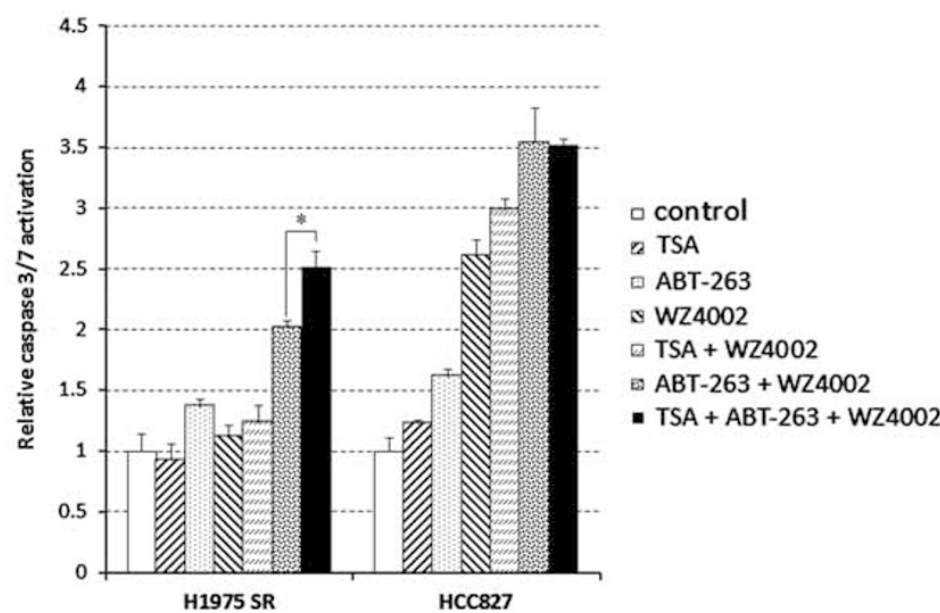

C

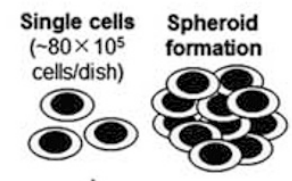

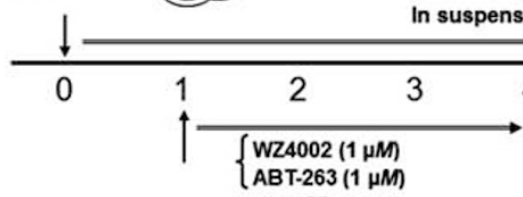
or $\left\{\begin{array}{l}\text { WZ4002 }(1 \mu M) \\ \text { ABT-263 }(1 \mu M)\end{array}\right.$ trichostatin $\mathrm{A}(0.1 \mu \mathrm{M})$

d
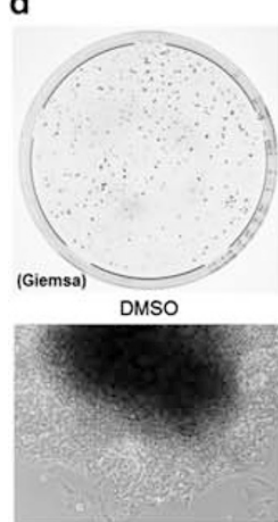

H1975 SR

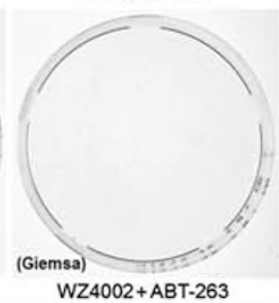

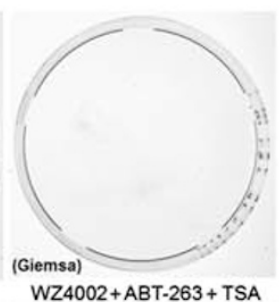

WZ4002+ABT-263+TSA

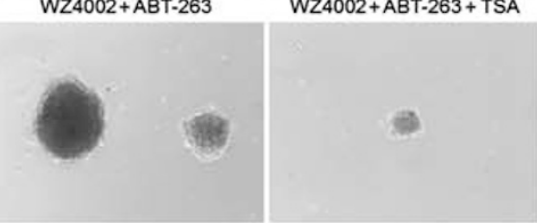

WZ4002 (1 $\mu M)$
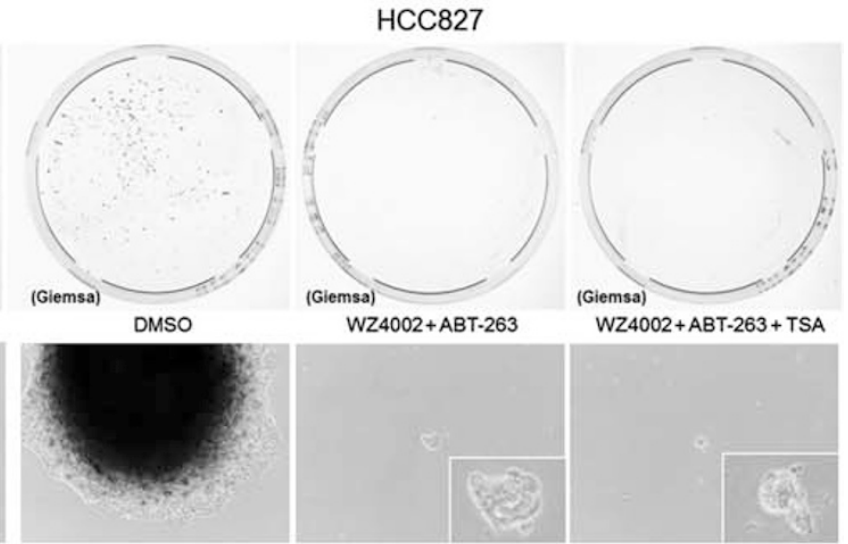

WZ4002+ABT-263 9 days

ABT-263 $(1 \mu M)$
trichostatin A $(0.1 \mu M)$

in suspension in suspension
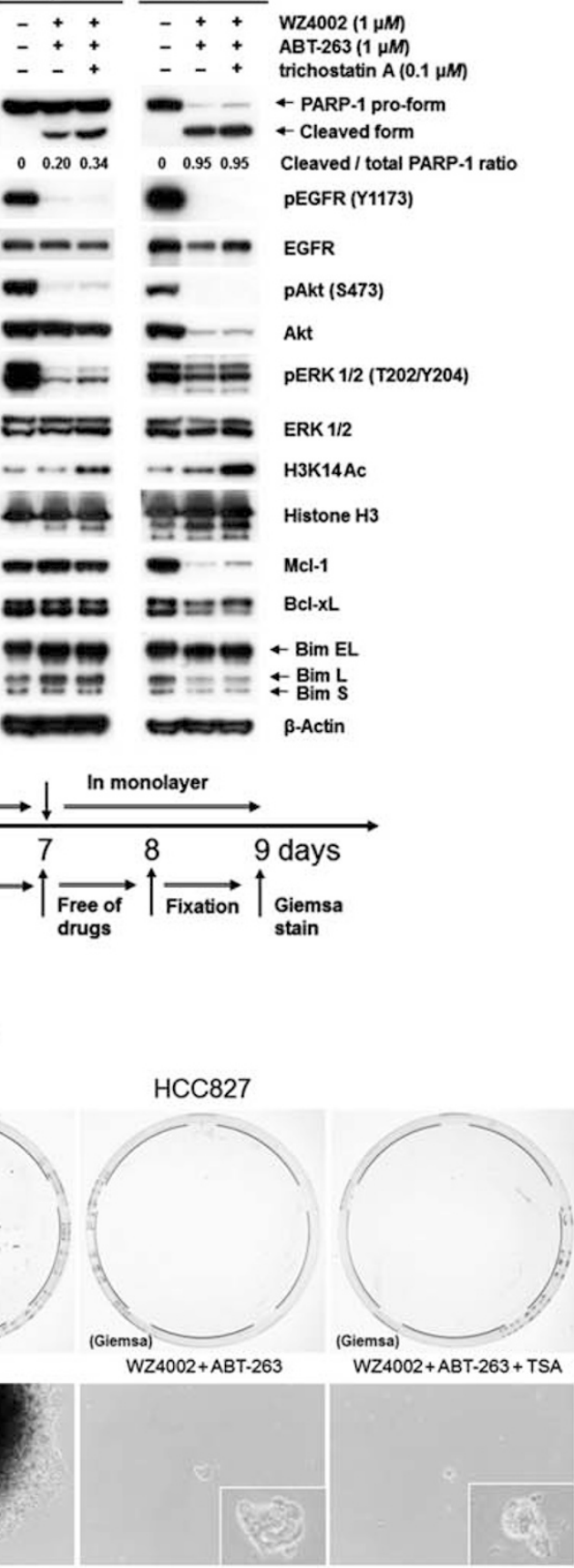

e

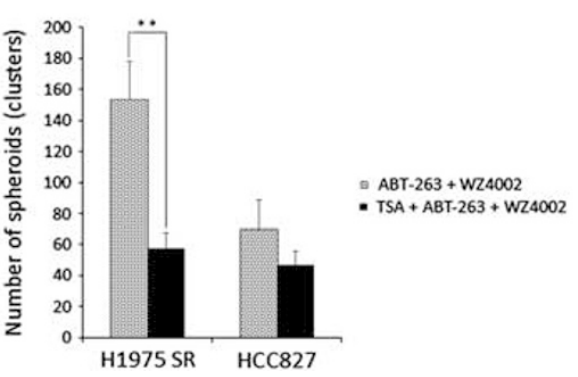


TSA similarly reduced the phosphorylation levels of EGFR, Akt, and ERK 1/2. The proapoptotic Bcl-2 family member Bim extra-long isoform appeared to be dephosphorylated in both the cell lines treated, judging from a faster migration in PAGE compared with control (Figure 6b). The dephosphorylated Bim extra-long isoform has a critical role in the induction of apoptosis in EGFR-mutant lung adenocarcinomas. ${ }^{9,10}$ Interestingly, the expression level of anti-apoptotic Bcl-2 family member Mcl-1 remained unaffected in H1975 SR cells when the cells were treated, whereas the Mcl-1 protein expression was markedly decreased in HCC827 cells treated, compared with untreated cells (Figure 6b). Slight downregulation of $\mathrm{Bcl}-\mathrm{xL}$ expression was also detected only in HCC827 cells, but not in H1975 SR cells when treated (Figure 6b). The decreased expression levels of Mcl-1 and Bcl-xL observed only in HCC827 cells may account for a considerable difference in apoptosis rates between treated H1975 SR cells and treated HCC827 cells. When H1975 SR cells in suspension were treated with the combination therapy in the presence or absence of TSA for $144 \mathrm{~h}$, the combination therapy including TSA significantly reduced the number of viable spheroids adhering to the conventional culture dish (WZ4002 + ABT-263 vs WZ4002 + ABT-263 + TSA: 154 vs $58, P=0.0083)$. The size of spheroids seemed to decrease after treatment with all the three drugs, compared with WZ4002 and ABT-263 treatment (Figure 6c-e). These findings suggest that H1975 SR cells have subpopulations that resist the combination treatment with WZ4002 and ABT-263, and the subpopulations, at least in part, are susceptible to HDAC inhibition. When suspended HCC827 cells were treated with the combined therapy with or without TSA for $144 \mathrm{~h}$, only small clusters of 10 or less cells, which appeared smaller in size than remaining spheroids of H1975 SR cells, were interspersed on the dish (Figure $6 \mathrm{c}$ and d). Interestingly, the number of HCC827 cell clusters remaining after longterm treatment with WZ4002, ABT-263, and TSA was a little smaller than that after treatment with WZ4002 and ABT-263 (WZ4002 + ABT-263 vs WZ4002 + ABT-263 + TSA: 70 vs 47, $P=0.1109$; Figure 6e), although the degree of apoptosis induced by the combination therapies with or without TSA were comparable in short-term $(24 \mathrm{~h})$ experiments (Figure 6a and b). Collectively, when suspended H1975 SR cells and HCC827 cells were treated with the combination therapy of WZ4002, ABT-263, and TSA for $144 \mathrm{~h}$, very few cells were able to survive the therapy and then adhere to the monolayer culture dishes in both cell lines.

\section{Intrasinus Floating Lung Adenocarcinoma Cells and Matrix-Adhesive Tumor Cells in Lymph Nodes Express a Mutant-Specific EGFR}

We examined metastasis-positive lymph nodes obtained from 16 EGFR-mutant lung adenocarcinoma patients for the expression of the mutant (E746-A750 deletion or L858R)specific EGFR (Table 1). Floating tumor cells in sinuses and/ or afferent lymphatic vessels were observed in all lymph nodes assessed. Intrasinus and/or intra-vessel floating lung adenocarcinoma cells in lymph nodes expressed mutantspecific EGFR in the same way as matrix-adhesive tumor cells (Table 1 and Figure 7).

\section{DISCUSSION}

Several laboratories, including ours, have reported that Src kinase activation has a role in the anoikis resistance in lung adenocarcinomas. ${ }^{3-5}$ Here, we have demonstrated that although long-term combination therapy that includes a Src TKI induces extensive apoptosis in suspended HCC827 cells to the point where they are almost eradicated, H1975 cells in suspension are clearly resistant to the therapy (Figure $2 \mathrm{~d}$ and e). These experimental findings suggest that Src TKIs may not always induce apoptosis effectively in EGFR-mutant lung adenocarcinoma cells floating in circulation or pleural effusion, although several phase II clinical trials with Src TKIs in patients with non-small cell lung cancer are currently underway. ${ }^{23,24}$ It has been reported that although cells such as H1975 cells, which express EGFR with L858R/T790M double mutations, resist Src TKIs, cells expressing EGFR with exon 19 deletion/T790M mutations remain sensitive to the same agents. ${ }^{14,15,25}$ Thus, whether Src TKIs are ineffective to all EGFR-mutant cells containing T790M should be examined in the future. HCC827 cells and H1975 cells, both of which have an activating EGFR mutation, depend on constitutive kinase activation of EGFR and its downstream signaling for survival; this dependence is called 'oncogene addiction..${ }^{11}$ Also, mutant EGFR and Src interact with each other. ${ }^{16}$ The interesting finding that the Src TKI AZD0530 considerably reduced the phosphorylation levels of Src and EGFR in HCC827 cells, although the EGFR phosphorylation in H1975 cells remained unaffected by this Src TKI, may account for the marked difference in the susceptibility to Src TKI-induced apoptosis between the two cell lines (Figure 1a). The third-generation EGFR TKI, WZ4002 similarly downregulated the phosphorylation levels of EGFR and its main downstream molecules, Akt and ERK 1/2, in H1975 SR cells, as well as HCC827 cells in suspension (Figure 6b). WZ4002 is 30- to 100 -fold more potent against the T790M EGFR mutation, and up to 100fold less potent against wild-type EGFR, compared with the other currently available EGFR TKIs. WZ4002 is a pyrimidine-based EGFR TKI, whereas all other EGFR TKIs are quinazoline-based TKIs. ${ }^{12,17}$ Consequently, WZ4002 is a promising drug in the treatment of EGFR-mutant lung adenocarcinomas.

Combination therapy of WZ4002 and ABT-263 was highly effective for HCC827 and H1975 SR cells in suspension. Intriguingly, the levels of apoptosis induced by the combination therapy were significantly higher in suspended cells than in adherent cells on conventional monolayer culture dishes for both cell lines (Figure $5 \mathrm{a}$ and $\mathrm{b}$ ). Such a markedly increased susceptibility to EGFR TKI-induced apoptosis in suspended conditions was also confirmed in gefitinib-treated HCC827 cells (Supplementary Figure S2a and b). To our 


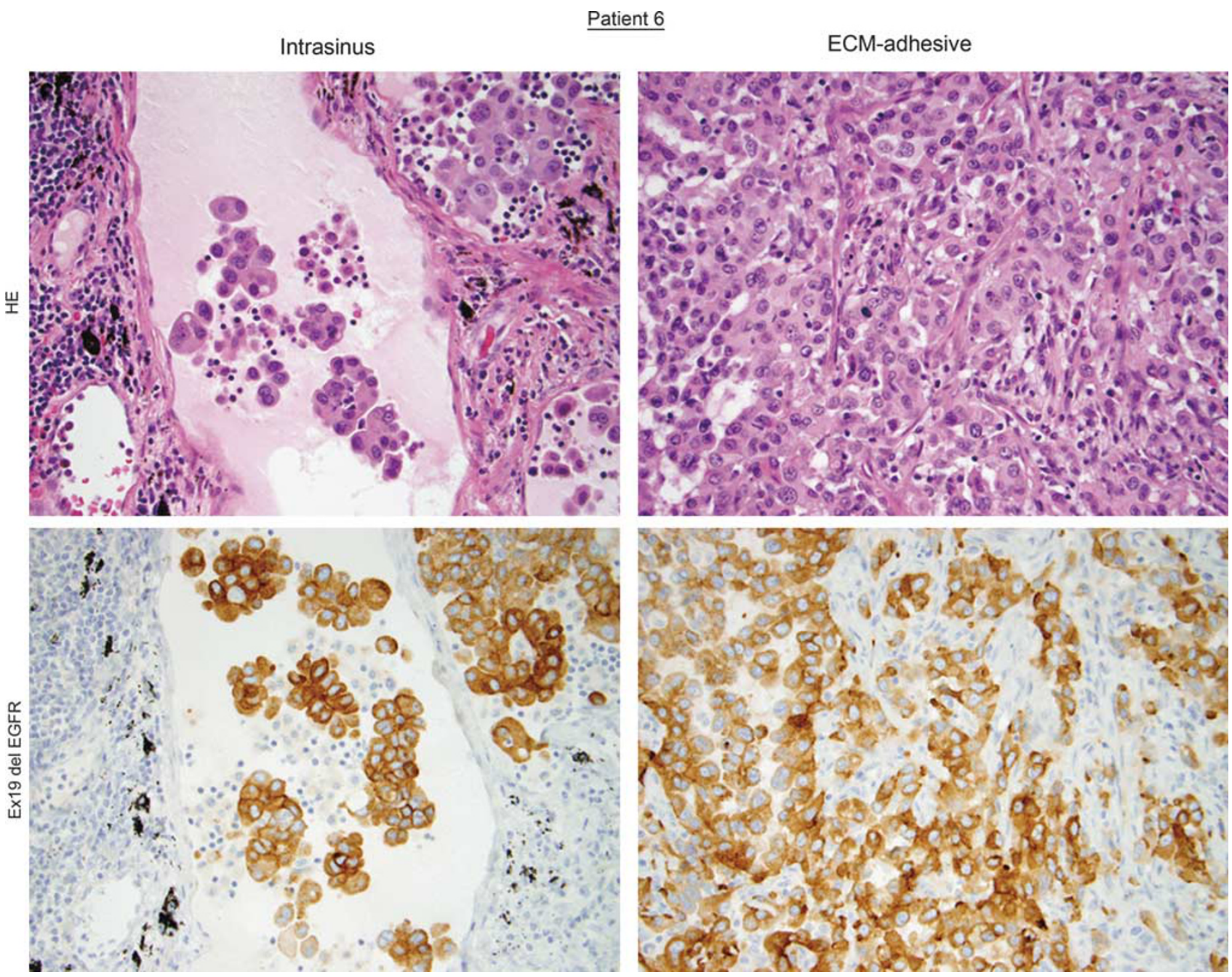

Figure 7 Intrasinus floating lung adenocarcinoma cells and matrix-adhesive tumor cells in lymph nodes express the mutant-specific (E746-A750 deletion) epidermal growth factor receptor (EGFR). Immunohistochemical analysis of a metastasis-positive lymph node obtained from a patient with EGFR-mutant lung adenocarcinoma.

knowledge, this is the first report to indicate that EGFRmutant lung adenocarcinoma cells depend critically on constitutive EGFR activation to thrive in anchorage-independent conditions. Attached EGFR-mutant lung adenocarcinoma cells appear less dependent on constitutive EGFR signaling for survival than detached cells, probably because integrin ligation enhances cell survival in adherent cells. ${ }^{26}$ These in vitro phenomena are supported by a clinically relevant case report that EGFR-mutant lung adenocarcinoma cells floating in pleural effusion in a patient actually underwent apoptosis to some extent as early as $4 \mathrm{~h}$ on gefitinib therapy. ${ }^{27}$

Recent articles have demonstrated that even established cancer cell lines are not homogeneous, and identified rare, slowly cycling subpopulations in lung, melanoma, breast, and colon cancer cell lines. ${ }^{22,28-30}$ During our manuscript preparation, subpopulations of HCC827 cells with a quiescence-like state that resist EGFR TKIs have been reported to be highly susceptible to ABT-737, the predecessor of
ABT-263. ${ }^{29}$ Moreover, a report showed that HDAC inhibitors synergistically enhanced the levels of EGFR TKI-induced apoptosis in EGFR-mutant lung adenocarcinoma cell lines. ${ }^{31}$ Therefore, it seems reasonable that suspended HCC827 cells were highly vulnerable to the combined treatment with WZ4002, ABT-263 with or without TSA, and consequently resulted in almost complete cell death by the long-term combination therapy (Figure 6d). On the other hand, H1975 SR cells are likely to have subpopulations that can evade apoptosis induced by the combination therapy of WZ4002 and ABT-263, and these subpopulations of cells seem to be susceptible to TSA (Figure 6d and e). Consequently, H1975 SR cells were highly sensitive to the combination therapy with WZ4002, ABT-263, and TSA, and this combination therapy effectively induced apoptosis in H1975 SR cells to the level of almost eradication after long-term treatment (Figure 6d). HDAC inhibitors have proapoptotic effects on quiescent cancer stem cells or slowly cycling cells that often resist 
therapy and serve as the source of relapse by reducing Wnt signaling, which has a critical role in stem cell maintenance, or by inhibiting the chaperone function of heat shock protein 90. ${ }^{13,31,32}$ Our results suggest that H1975 SR cells have slowly growing subpopulations that are sensitive to HDAC inhibitors. However, the molecular mechanisms underlying the proapoptotic effect of TSA on these subpopulations in H1975 SR cells remain to be elucidated.

This study reveals that floating EGFR-mutant lung adenocarcinoma cells in sinuses and/or afferent lymphatic vessels in all lymph nodes examined expressed a mutant-specific EGFR (Figure 7). Thus, we expect that the combination therapy of WZ4002, ABT-263, and TSA as components of adjuvant chemotherapy for patients with vessel invasion-positive $E G F R$-mutant lung adenocarcinoma could induce massive apoptosis in intra-vessel circulating tumor cells and consequently decrease the development of metastases. Unfortunately, we did not confirm the in vivo effectiveness of the combination therapy, because both H1975 SR and HCC827 cells $\left(1 \times 10^{6}\right.$ cells each) injected into $\mathrm{BALB} / \mathrm{c}$ nude mice via the tail vein did not produce pulmonary metastatic lesions of macroscopic size, even 8 weeks after the inoculation using the experimental lung metastasis assay (data not shown).

In conclusion, the findings presented here suggest that $\mathrm{Src}$ TKIs cannot completely overcome the anoikis resistance in lung adenocarcinoma with the EGFR T790M mutation. The suspended EGFR-mutant lung adenocarcinoma cell lines examined are highly susceptible to the combination therapy of WZ4002, ABT-263, and TSA. This suggests that EGFRmutant adenocarcinoma cells depend critically on constitutive EGFR activation to thrive in anchorage-independent (ie, suspended) conditions. Moreover, we confirmed that floating EGFR-mutant lung adenocarcinoma cells in sinuses and/or afferent lymphatic vessels in lymph nodes actually express the mutant-specific EGFR. Collectively, these results suggest that EGFR TKIs can induce apoptosis more effectively in EGFR-mutant lung adenocarcinoma cells floating in circulation or pleural effusion than in an extracellular matrixadherent state in vivo. As vessel-invasion positive lung adenocarcinoma patients frequently experience relapse after surgery, the establishment of effective adjuvant chemotherapy for these patients is urgently needed. We expect that patients with EGFR-mutant lung adenocarcinoma would benefit from the combination therapy of WZ4002, ABT-263, and TSA. This combination therapy could induce apoptosis effectively in circulating tumor cells and reduce the development of distant metastases.

\section{ACKNOWLEDGEMENTS}

This work was supported in part by a Grant-in-Aid for Young Scientists (B) (22790367) from the Ministry of Education, Culture, Sports, Science, and Technology of Japan (Y Sakuma)

\section{DISCLOSURE/CONFLICT OF INTEREST}

The authors declare no conflict of interest.
Supplementary Information accompanies the paper on the Laboratory Investigation website (http://www.laboratoryinvestigation.org)

1. Sakuma $Y$, Okamoto $N$, Saito $H$, et al. A logistic regression predictive model and the outcome of patients with resected lung adenocarcinoma of $2 \mathrm{~cm}$ or less in size. Lung Cancer 2009;65:85-90.

2. Frisch $\mathrm{SM}$, Francis $\mathrm{H}$. Disruption of epithelial cell-matrix interactions induces apoptosis. J Cell Biol 1994;124:619-626.

3. Wei $L$, Yang $Y$, Zhang $X$, et al. Altered regulation of Src upon cell detachment protects human lung adenocarcinoma cells from anoikis. Oncogene 2004;23:9052-9061.

4. Uekita T, Jia L, Narisawa-Saito M, et al. CUB domain-containing protein 1 is a novel regulator of anoikis resistance in lung adenocarcinoma. Mol Cell Biol 2007;27:7649-7660.

5. Sakuma Y, Takeuchi T, Nakamura Y, et al. Lung adenocarcinoma cells floating in lymphatic vessels resist anoikis by expressing phosphorylated Src. J Pathol 2010;220:574-585.

6. Tse C, Shoemaker AR, Adickes J, et al. ABT-263: a potent and orally bioavailable Bcl-2 family inhibitor. Cancer Res 2008;68:3421-3428.

7. Cragg MS, Harris $C$, Strasser A, et al. Unleashing the power of inhibitors of oncogenic kinases through BH3 mimetics. Nat Rev Cancer 2009;9:321-326.

8. Sakuma Y, Tsunezumi J, Nakamura Y, et al. ABT-263, a Bcl-2 inhibitor, enhances the susceptibility of lung adenocarcinoma cells treated with Src inhibitors to anoikis. Oncol Rep 2011;25:661-667.

9. Cragg MS, Kuroda J, Puthalakath $\mathrm{H}$, et al. Gefitinib-induced killing of NSCLC cell lines expressing mutant EGFR requires BIM and can be enhanced by BH3 mimetics. PLoS Med 2007;4:1681-1689.

10. Gong $Y$, Somwar R, Politi $K$, et al. Induction of BIM is essential for apoptosis triggered by EGFR kinase inhibitors in mutant EGFRdependent lung adenocarcinomas. PLoS Med 2007;4:e294.

11. Sharma SV, Bell DW, Settleman J, et al. Epidermal growth factor receptor mutations in lung cancer. Nat Rev Cancer 2007;7:169-181.

12. Pao W, Chmielecki J. Rational, biologically based treatment of EGFRmutant non-small-cell lung cancer. Nat Rev Cancer 2010;10:760-774.

13. Zhang $B$, Strauss $A C$, Chu $S$, et al. Effective targeting of quiescent chronic myelogenous leukemia stem cells by histone deacetylase inhibitors in combination with imatinib mesylate. Cancer Cell 2010;17: 427-442.

14. Song $L$, Morris $M$, Bagui $T$, et al. Dasatinib (BMS-354825) selectively induces apoptosis in lung cancer cells dependent on epidermal growth factor receptor signaling for survival. Cancer Res 2006;66: 5542-5548.

15. Zhang J, Kalyankrishna S, Wislez $M$, et al. SRC-family kinases are activated in non-small cell lung cancer and promote the survival of epidermal growth factor receptor-dependent cell lines. Am J Pathol 2007;170:366-376

16. Chung BM, Dimri M, George M, et al. The role of cooperativity with Src in oncogenic transformation mediated by non-small cell lung cancerassociated EGF receptor mutants. Oncogene 2009;28:1821-1832.

17. Zhou W, Ercan D, Chen L, et al. Novel mutant-selective EGFR kinase inhibitors against EGFR T790M. Nature 2009;462:1070-1074.

18. Soldatenkov VA, Smulson M. Poly(ADP-ribose) polymerase in DNA damage-response pathway: implications for radiation oncology. Int J Cancer 2000;90:59-67.

19. Mansy SS. Agarose cell block: innovated technique for the processing of urine cytology for electron microscopy examination. Ultrastruct Pathol 2004;28:15-21.

20. Matsukuma S, Yoshihara M, Kasai F, et al. Rapid and simple detection of hot spot point mutations of epidermal growth factor receptor, BRAF, and NRAS in cancers using the loop-hybrid mobility shift assay. J Mol Diagn 2006;8:504-512.

21. Yao Z, Fenoglio S, Gao DC, et al. TGF-beta IL-6 axis mediates selective and adaptive mechanisms of resistance to molecular targeted therapy in lung cancer. Proc Natl Acad Sci USA 2010;107:15535-15540.

22. Sharma SV, Lee DY, Li B, et al. A chromatin-mediated reversible drug-tolerant state in cancer cell subpopulations. Cell 2010;141:69-80.

23. Kim LC, Song L, Haura EB. Src kinases as therapeutic targets for cancer. Nat Rev Clin Oncol 2009;6:587-595.

24. Haura EB, Tanvetyanon T, Chiappori A, et al. Phase I/II study of the Src inhibitor dasatinib in combination with erlotinib in advanced non-small-cell lung cancer. J Clin Oncol 2010;28:1387-1394. 
25. Fu YN, Yeh $\mathrm{CL}$, Cheng $\mathrm{HH}$, et al. EGFR mutants found in non-small cell lung cancer show different levels of sensitivity to suppression of Src: implications in targeting therapy. Oncogene 2008;27:957-965.

26. Desgrosellier JS, Cheresh DA. Integrins in cancer: biological implications and therapeutic opportunities. Nat Rev Cancer 2010;10:9-22.

27. Kubo $A, K o h Y, K a w a g u c h i ~ T$, et al. Malignant pleural effusion from lung adenocarcinoma treated by gefitinib. Intern Med 2011;50: 745-748.

28. Roesch A, Fukunaga-Kalabis M, Schmidt EC, et al. A temporarily distinct subpopulation of slow-cycling melanoma cells is required for continuous tumor growth. Cell 2010;141:583-594.
29. Fan W, Tang Z, Yin L, et al. MET-independent lung cancer cells evading EGFR kinase inhibitors are therapeutically susceptible to $\mathrm{BH} 3$ mimetic agents. Cancer Res 2011;71:4494-4505.

30. Dey-Guha I, Wolfer A, Yeh AC, et al. Asymmetric cancer cell division regulated by AKT. Proc Natl Acad Sci USA 2011;108:12845-12850.

31. Edwards A, Li J, Atadja P, et al. Effect of the histone deacetylase inhibitor LBH589 against epidermal growth factor receptor-dependent human lung cancer cells. Mol Cancer Ther 2007;6:2515-2524.

32. Burgess $\mathrm{A}$, Ruefli $\mathrm{A}$, Beamish $\mathrm{H}$, et al. Histone deacetylase inhibitors specifically kill nonproliferating tumour cells. Oncogene 2004;23: 6693-6701. 